\title{
The mechanical and material properties of elderly human articular cartilage subject to impact and slow loading
}

Running title: Impact loading and composition of human cartilage

L.V. Burgin ${ }^{1}$, L. Edelsten ${ }^{2}$ and R.M. Aspden*

Musculoskeletal Research Programme, School of Medicine \& Dentistry, University of Aberdeen, Aberdeen, UK.

${ }^{1}$ Current address: Medical Technologies Innovation and Knowledge Centre, X102 Medical and Biological Engineering - University of Leeds, Leeds, LS2 9JT. UK.

L.V.Burgin@leeds.ac.uk

${ }^{2}$ Current email address: Lorna.ramsay@education.gsi.gov.uk

\section{Corresponding Author}

Professor R.M. Aspden

Musculoskeletal Research Programme

School of Medicine \& Dentistry

University of Aberdeen

Institute of Medical Sciences

Foresterhill

Aberdeen AB25 2ZD

UK

Tel: + (0)1224 437445

e-mail: r.aspden@abdn.ac.uk 


\begin{abstract}
The mechanical properties of articular cartilage vary enormously with loading rate, and how these properties derive from the composition and structure of the tissue is still unclear. This study investigates the mechanical properties of human articular cartilage at rapid rates of loading, compares these with measurements at slow rates of loading and explores how they relate to the gross composition of the tissue. Full-depth femoral head cartilage biopsies were subjected to a slow, unconfined compression test followed by an impact at an energy of 78.5 $\mathrm{mJ}$ and velocity $1.25 \mathrm{~m} \mathrm{~s}^{-1}$. . The modulus was calculated from the slope of the loading curve and the coefficient of restitution from the areas under the loading and unloading curves. Tissue composition was measured as water, collagen and glycosaminoglycan contents. The maximum dynamic modulus ranged from 25 to $150 \mathrm{MPa}$. These values compared with 1-3 MPa measured during quasi-static loading. The coefficient of restitution was 0.502 (0.066) (Mean (Standard deviation)) and showed no site variation. Water loss was not detectable. Composition was not strongly associated with modulus; water and collagen contents together predicted about $25 \%$ of the variance in modulus.
\end{abstract}

Keywords: articular cartilage; human; femoral head, impact; mechanical properties; composition; material properties

Subjects areas: Biomechanics, Biomaterials 


\section{Introduction}

Articular cartilage provides a resilient, low-friction bearing surface to bones where they articulate in diarthrodial joints. It is a remarkably smooth and tough material and protects the joint by distributing applied loads. Mechanical damage caused, for instance, by trauma may lead to osteoarthritis (OA) and studies in vivo have suggested that cell damage, oxidative stress and apoptosis may play a significant role in this process [1,2]. The chondrocytes are protected by being embedded in an extensive matrix that has a complicated structure. They can regulate their biosynthesis of matrix molecules, and thereby control the composition of the matrix, in order to match the mechanical properties of the tissue to the prevailing loading environment, but their regenerative capacity is limited. Relationships between the composition and structure of the tissue and its mechanical properties are still poorly understood and the advent and rapid expansion of attempts to engineer replacement articular cartilage make understanding this relationship increasingly urgent as efforts are made to match the repair tissue to the natural tissue [3]. Measurements of the mechanical properties depend strongly on the rate of loading [4] and the nature of the test performed, for example, confined or unconfined. In addition, many studies have used animal, especially bovine, tissue but it is becoming apparent that bovine tissue is not a good model for human tissue, metabolically, structurally or mechanically [5-9].

We have hypothesized that cartilage is a biological example of a fibre-composite material in which the strong and stiff collagen fibrils reinforce a mechanically weak proteoglycan gel [10]. In such materials interactions between components play an important role [11]. In a previous study of bovine cartilage, in which we found the site variation of composition over the tibial plateau to be similar to previous reports from other joints, we found no clear relationship between gross composition and modulus measured at slow rates of loading [12] 
There are few studies relating tissue composition to mechanical properties at high rates of loading. Confined compression tests on bovine tissue using a porous impactor $[13,14]$ reported moduli of over $1000 \mathrm{MPa}$, dependent on stress rate and proteoglycan and water contents. Water loss increased with stress but decreased with stress rate and the relationship between proteoglycan content and dynamic modulus was reported to be weak [14]. In our previous studies, however, no significant water loss was detected from bovine cartilage subjected to impact loads [15].

Early studies of the mechanical properties of cartilage subjected to impact loads reported that, per unit thickness, cartilage attenuated peak forces ten-times more effectively than bone $[16,17]$. Using a drop tower to apply an impact load to achieve strains of $10-50 \%$ at strain rates of $500 \mathrm{~s}^{-1}$ or $1000 \mathrm{~s}^{-1}$ Finlay and Repo [18] found stresses of $25 \mathrm{MPa}$ were required to produce structural damage or chondrocyte death in human tissue. These stresses corresponded to strains of approximately $20-30 \%$ and involved energy absorption of $1 \mathrm{MJ} \mathrm{m}^{-3}$. No difference was found between the moduli at the two strain rates [19]. In subsequent studies, however, using a wider range of strain-rates in unconfined compression testing, moduli have been reported up to several hundred megaPascals with a dependency both on stress- or strainrate and stress magnitude (summarized by Natoli and Athanasiou [20]). Oloyede et al. [21], using a pendulum device and bovine cartilage, showed that the compressive modulus at an applied stress of $0.5 \mathrm{MPa}$ increased linearly as the strain rate increased from $5 \times 10^{-5}$ to $5 \times 10^{-3}$ $\mathrm{s}^{-1}$ before reaching a limit of approximately $20 \mathrm{MPa}$. The applied stress was much less than typical physiological stresses and the J-shaped stress-strain curve would lead to a correspondingly lower measured modulus. At greater stresses, in unconfined compression testing of bovine cartilage using a drop tower, we found values for the maximum modulus of up to approximately $300 \mathrm{MPa}$, occurring at stresses of the order of $80 \mathrm{MPa}$. Thresholds for 
applied stress, stain-rate and energy that resulted in permanent damage to the tissue were identified by Verteramo and Seedhom [22] by measuring the viscoelastic storage and loss moduli in bovine cartilage following an impact load. They found changes in the mechanical properties following stresses above $25 \mathrm{MPa}$, similar to those found previously, with the energy absorbed per unit volume of $12.79 \mathrm{MJ} \mathrm{m}^{-3}$. Differences have been reported, however, between human and bovine tissue subjected to impacts of equal energy using both an impactor, in which the whole tissue is loaded, and an indenter in which only a central core is subjected to direct loading. Higher modulus values were found in bovine tissue than human [5]. Some of the differences may be due to different thicknesses of tissue but differences in failure behaviour and modulus were more difficult to explain. Others found differences between human and bovine, albeit at slower loading rates, with moduli reported of about 1.6 MPa for human tissue and no attempt to relate mechanical measurements to composition [9].

The aim of this study was to measure the mechanical properties of elderly articular cartilage during impact loading, compare these data with similar measurements recorded from the same tissue under slow loading and investigate how these are related to collagen, glycosaminoglycan (GAG), and water contents of the tissue. To do this, full-depth cores of cartilage were taken from defined sites over the human femoral head. These data will provide a framework for comparison with biosynthetic constructs.

\section{Materials and methods}

\subsection{Tissue preparation}

Human femoral heads were obtained following a hemi-arthroplasty for a fracture of the neck of the femur (eight male, six female, average age 79 years, age range 63-89 years). Approval was obtained for the use of this material from the Local Research Ethics Committee. Only femoral heads with visual evidence of minimal cartilage fibrillation were used. Full-depth 
cartilage samples with no bone, $5 \mathrm{~mm}$ in diameter, were removed from eleven sites over the femoral head using a cork borer and scalpel [12]. To ensure repeatability of sample sites, a master template was created (Fig. 1) and copied to ultrasound probe covers (SSL International plc, Knutsford) that are stretchy and semi-transparent. The template markings were then transferred to the cartilage by injecting a small spot of bromophenol blue through the probe cover into the femoral head. The supero-anterior (SA) and supero-posterior (SP) samples were at $30^{\circ}$ from the most superior site (S) to encompass the region of peak loading [23]. Samples were maintained in phosphate-buffered saline (PBS) except during the testing procedures.

The thickness of each sample was measured optically using a Zeiss Stemi-2000 stereomicroscope and AxioVision software (Zeiss Ltd, Welwyn Garden City) calibrated with a graticule slide, marked with $0.01 \mathrm{~mm}$ divisions. Samples were placed on their side and the thickness measured perpendicular to the articular surface at several locations around the perimeter. Any samples that were measurably wedge-shaped, more than $0.05 \mathrm{~mm}$ difference
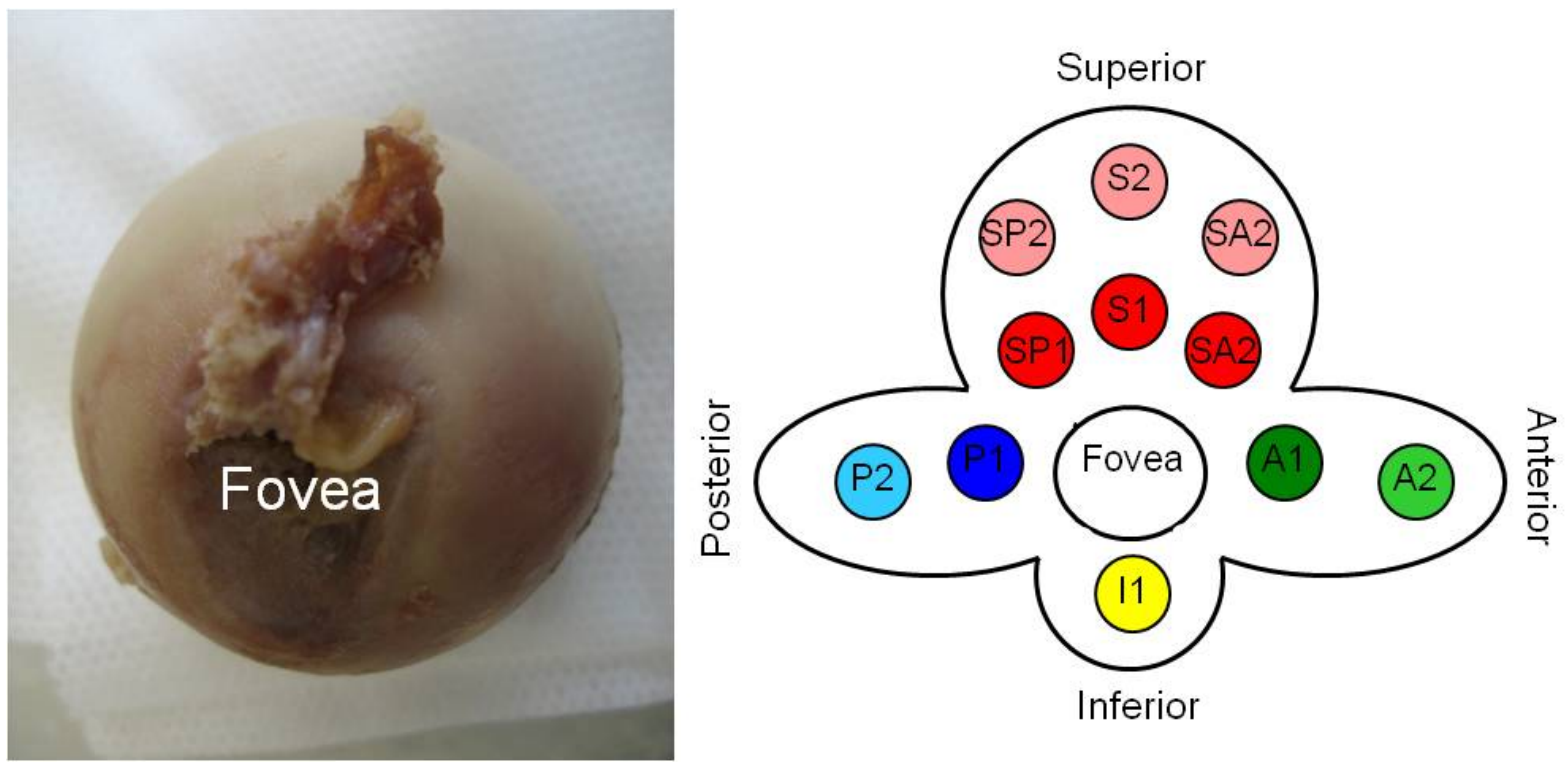

Figure 1Human femoral head and schematic diagram to show sites and nomenclature for cartilage removal. The fovea is the site of attachment of the ligamentum teres. The superoanterior (SA) and supero-posterior (SP) samples were located at $30^{\circ}$ from the most superior site $(\mathrm{S})$ to encompass the region of peak loading. 
across a sample, were removed at this stage. Excess fluid was removed from the surface by gentle blotting between moistened paper tissues and each sample was then weighed in a preweighed Eppendorf tube containing 1 mL of PBS. Each biopsy was weighed three times: just prior to testing (one day after sample removal), immediately after the impact test and a third time 24 hours later in order to measure how much water was lost or gained. In previous studies we have found that the tissue mass equilibrates within 24 hours of removal from the joint [24]. Each sample was tested first in slow compression, equilibrated for at least 30 minutes in PBS and then subjected to an impact test as described below.

\subsection{Slow Compression Testing}

Unconfined compression testing was done using an Instron materials testing machine, model 5564, fitted with a $10 \mathrm{~N}$ load cell (Instron Ltd, High Wycombe). The cross-head speed was $100 \%$ strain $\min ^{-1}\left(0.0167 \mathrm{~s}^{-1}\right)$. The peak stress was limited to $0.15 \mathrm{MPa}$ in order not to damage the tissue samples prior to impact loading. This was tested in pilot studies. A small metal plate and ball bearing between the cartilage and load cell ensured uniform loading of the sample. A second order polynomial was fitted to the stress-strain curve and the modulus, $E_{\text {slow }}$, found by differentiating the curve (Origin Software, Version 6.1; Aston Scientific Ltd, Stoke Mandeville) at a stress of 0.1 MPa.

\subsection{Impact Testing}

The drop tower and instrumentation used for impact testing have been described in detail elsewhere [25]. Briefly, each sample was placed on a stainless steel plate on top of a piezoelectric force transducer (Kistler Instruments Ltd., Alton). An impactor, with a mass of $100 \mathrm{~g}$, was released mechanically from a height of $80 \mathrm{~mm}$ above the articular surface and fell freely onto the sample. This corresponded to a calculated impact energy of $78.5 \mathrm{~mJ}$ and a 
contact velocity of $1.25 \mathrm{~m} \mathrm{~s}^{-1}$. The impactor was fitted with an accelerometer (Kistler Instruments Ltd., Alton), capable of measuring accelerations up to $500 \mathrm{~g}$, where $g$ is the acceleration due to gravity. These values were chosen based on our previous studies in order to produce minimal damage to the tissue.

Data from the force transducer and the accelerometer were low-pass filtered and the resulting curve overlaid on the original to check that no distortion had occurred. Force data were converted to engineering stress by dividing by the original cross-sectional area of the sample. Accelerometer data were integrated twice to find the displacement and this was divided by the original thickness of the sample to determine the engineering strain. The stress-strain curve for each sample was then differentiated to obtain the modulus. The peak dynamic modulus, $E_{d y n}$, was found as the maximum gradient and, for comparison with slow loading, the modulus at $0.1 \mathrm{MPa}\left(E_{0.1}\right)$ from the gradient at this stress. Mean stress- and strain-rates were calculated from the peak value divided by the duration of impact. Peak strain-rate was calculated from the quotient of impact velocity and sample thickness, peak stress-rate from the stress-time data.

The energy of the applied impact was calculated from the mass and drop height, assuming a frictionless drop [24]. A force-displacement plot was produced for loading and unloading phases. The energy of deformation, $W_{c}$, was found from the area under the loading curve to maximum deformation and the energy released during restitution, $W_{r}$, by integrating the unloading curve from this point back to zero strain [15]. The square of the energetic coefficient of restitution is given by $e_{*}^{2}=-W_{r} / W_{c}[26]$ so that a perfectly elastic collision has $e_{*}=1$ and a plastic collision in which all the kinetic energy is dissipated has $e_{*}=0$. 


\subsection{Biochemical analysis}

The gross composition of the human femoral head cartilage samples was measured in terms of the mass of water, sulphated glycosaminoglycans and collagen as a percentage of total wet mass. Water content was measured by weighing before and after lyophilisation for 24 hours. Samples were then digested in $20 \mu \mathrm{L}$ x (cartilage wet mass in $\mathrm{mg}$ ) of papain solution (135 $\mu \mathrm{g}$ $\mathrm{mL}^{-1}$ containing $0.1 \mathrm{M}$ sodium acetate, $5 \mathrm{mM}$ EDTA and $5 \mathrm{mM}$ cysteine-HCl, $\mathrm{pH}$ 6.0) for 24 $\mathrm{h}$ at $65{ }^{\circ} \mathrm{C}$. Sulphated glycosaminoglycans were measured using a dimethylmethylene blue (DMMB) method, modified from that described by Stone et al. [27] for use in a 96-well plate, and standardised against chondroitin-6-sulphate (Sigma, C4384). Collagen content was measured using a hydroxyproline assay [28] assuming $13 \mathrm{~g}$ hydroxyproline per $100 \mathrm{~g}$ collagen [29].

\subsection{Statistical analysis}

Statistical analysis was done using SigmaStat 2.03 (SPSS UK Ltd, Woking). Data were tested for normality using the Kolmogorov-Smirnov test with the significance level set to $P=0.05$. Normally distributed data are presented as mean and standard deviation (mean (sd)), otherwise values are shown as median [25\%, 75\%] percentiles. Site variation was investigated using analysis of variance (ANOVA) with the null hypothesis being that there were no differences between sites. Pair-wise multiple comparisons were done using the Tukey test. If the data were not normally distributed then a Kruskal-Wallis ANOVA on ranks was performed instead and pair-wise comparisons performed using Dunn's method. The strength of association between parameters was calculated using Spearman's rank correlation. The contribution of composition to the mechanical properties was investigated using best-subsets regression, and linear regression was used to assess dependencies. 


\section{Results}

A number of samples were uneven or wedge-shaped, making testing unreliable, or were ejected from between the anvils during testing, resulting in 113 samples for analysis. The mean thickness of the cartilage was $1.42(0.45) \mathrm{mm}$ but significant variation was found over the femoral head $(P<0.001)$, ranging from $0.59-2.29 \mathrm{~mm}$. The thickest cartilage was found in an arc over the superior region (Table 1) and the thinnest cartilage below the fovea in the inferior and lower posterior and anterior regions. No damage was apparent to any of the samples and this was confirmed in histological sections from a random selection of impacted samples.

\subsection{Cartilage Modulus}

The stress-strain relationship was non-linear for both slow and impact loading; the modulus increased with increasing stress (Fig. 2). The dynamic and quasistatic moduli are given for each site in Table 1. $E_{d y n}$ varied significantly with location on the femoral head $(P<0.001)$ but correlated negatively with the thickness of the cartilage, $E_{d y n}=91.7-22.5$ [thickness] ( $R$ $=-0.49, P<0.001)$. Cartilage thickness will affect the strain rate and if $E_{d y n}$ was adjusted as if to unit thickness using the above regression the variation with site became non-significant $(P$ $=0.10)$. There was some site variation of $E_{\text {slow }}(P=0.055)$ with the greatest values for $E_{\text {slow }}$ found over the superior aspect and the inferior region having the smallest modulus. Correlation with thickness in this case was positive $(R=0.68, P<0.001)$. Values for the dynamic modulus at $0.1 \mathrm{MPa}, E_{0.1}$, were very similar to those for $E_{\text {slow }}$ (Table 1 ). The strain in the tissue at which $E_{d y n}$ occurred did not vary with site over the femoral head, $\operatorname{strain}\left(E_{d y n}\right)=$ $0.54(0.12)(P=0.52)$. 
Table 1. Site variation of cartilage thickness and moduli. Values shown are mean (SD) for peak dynamic modulus, $E_{d y n}$, the quasistatic modulus at $0.1 \mathrm{MPa}, E_{\text {slow }}$, and the dynamic modulus at $0.1 \mathrm{MPa}, E_{0.1}$, for samples at various sites over the human femoral head described in Figure 1.

\begin{tabular}{l|c|c|c|c} 
Site & $\begin{array}{c}\text { Thickness } \\
(\mathrm{mm})\end{array}$ & $\begin{array}{c}E_{\text {dyn }} \\
(\mathrm{MPa})\end{array}$ & $\begin{array}{c}E_{\text {slow }}(\mathrm{MPa}) \\
(\text { at } 0.1 \mathrm{MPa})\end{array}$ & $\begin{array}{c}E_{0.1}(\mathrm{MPa}) \\
\text { (at } 0.1 \mathrm{MPa})\end{array}$ \\
\hline $\mathrm{S} 1$ & $1.78(0.44)$ & $51.9(11.8)$ & $2.39(0.93)$ & $2.64(0.92)$ \\
S2 & $1.36(0.41)$ & $68.0(17.3)$ & $2.06(0.62)$ & $2.27(0.98)$ \\
SP1 & $1.53(0.36)$ & $53.6(10.8)$ & $2.22(0.48)$ & $2.36(0.85)$ \\
SP2 & $1.24(0.22)$ & $68.1(16.1)$ & $1.62(0.46)$ & $2.33(1.34)$ \\
SA1 & $1.72(0.47)$ & $54.2(7.9)$ & $2.47(0.49)$ & $2.49(0.89)$ \\
SA2 & $1.34(0.38)$ & $65.8(22.2)$ & $1.60(0.41)$ & $1.94(1.08)$ \\
P1 & $1.45(0.41)$ & $63.5(21.4)$ & $2.02(0.43)$ & $2.20(1.26)$ \\
P2 & $1.08(0.30)$ & $89.8(31.0)$ & $1.62(0.39)$ & $1.91(1.28)$ \\
A1 & $1.60(0.41)$ & $58.9(12.7)$ & $2.04(0.34)$ & $2.65(2.16)$ \\
A2 & $1.03(0.27)$ & $86.3(25.1)$ & $1.96(0.27)$ & $1.81(0.62)$ \\
I1 & $1.13(0.33)$ & $89.0(10.8)$ & $1.60(0.51)$ & $1.91(0.72)$ \\
\hline
\end{tabular}

Peak and mean stress-rates were strongly correlated $(R=0.98, P<0.001)$, as were peak and mean strain-rates $(R=0.97, P<0.001)$. There was a significant and strong correlation between $E_{d y n}$ and peak stress rate $(R=0.83, P<0.001)$, but a less strong correlation between $E_{d y n}$ and peak strain rate $(R=0.45, P<0.001)$. Peak stress-rates varied from $11.3 \mathrm{GPa} \mathrm{s}^{-1}$ to 130.7 $\mathrm{GPa} \mathrm{s}^{-1}$ and linear regression showed that $\log \left(E_{\text {dyn }}\right)=0.52 \log ($ peak stress rate $)+$ 2.22, $\left(R^{2}=0.76, P<0.001\right)$. Residuals were not normally distributed for the untransformed 

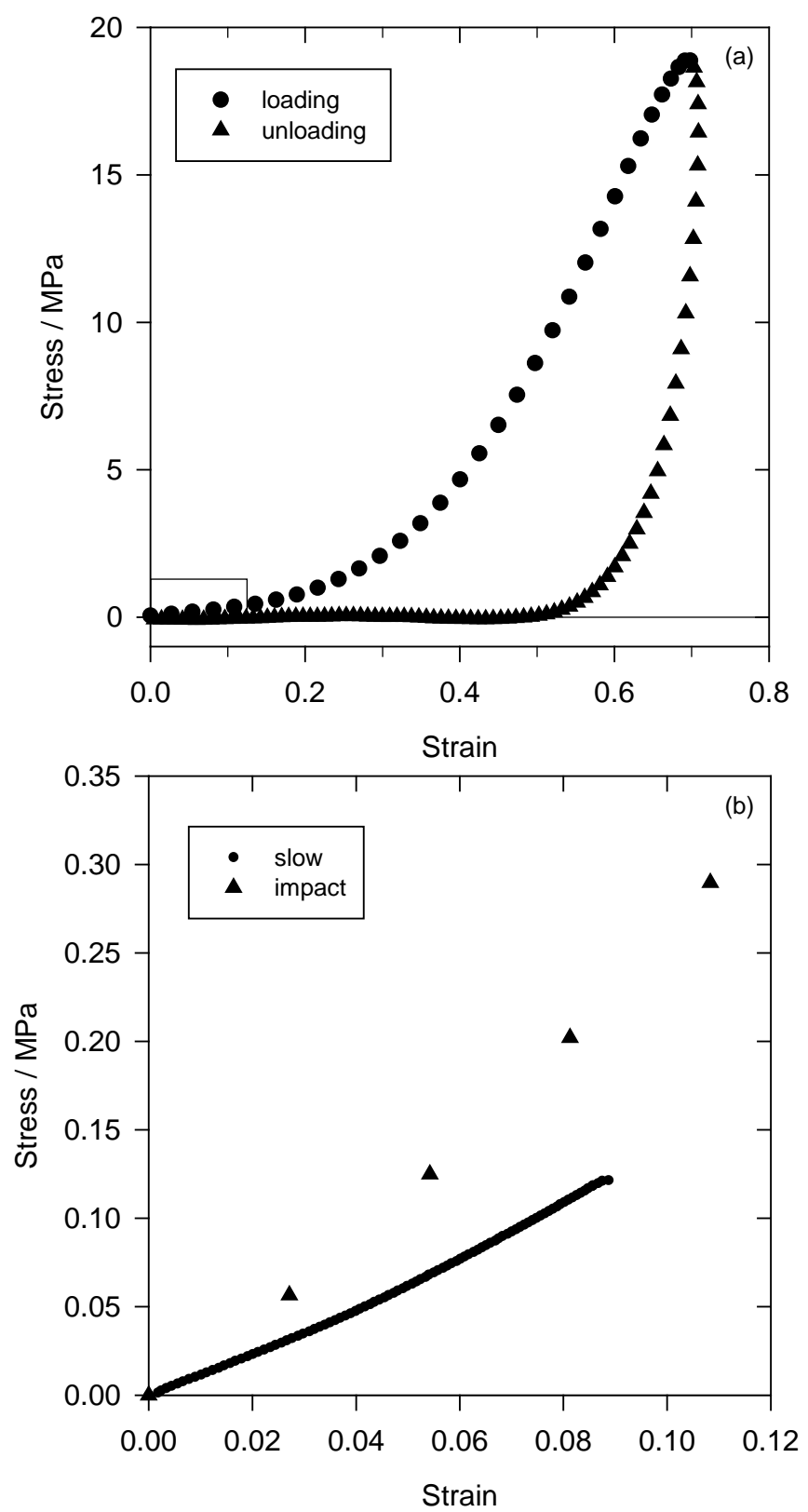

Figure 2 Typical stress strain curves from one sample of articular cartilage subjected to (a) an impact load and (b) slow loading. For comparison, the first few points of the impact curve are shown in (b) together with the curve from slow loading and the box in (a) shows approximately the corresponding region during impact. The energy stored and released is found by integrating to find the areas under the loading part of the curve to peak stress and the unloading part respectively, from which the coefficient of restitution is calculated

data but the logarithmic transform was well-behaved. Peak strain rates ranged between $492 \mathrm{~s}^{-1}$ in the thickest samples to $1854 \mathrm{~s}^{-1}$ in the thinnest. 


\subsection{Coefficient of restitution}

The mean energy of deformation of the cartilage samples during the impact process was calculated to be $75.5(1.8) \mathrm{mJ}$ and was strongly correlated with both cartilage thickness $(R=$ 0.78, $P<0.001)$ and negatively with $E_{d y n}(R=-0.77, P<0.001)$. Assuming a frictionless drop, this varied between 93-98 \% of the theoretical impact energy found from the height and mass of the impactor $(78.5 \mathrm{~mJ})$. Normalized for tissue volume the mean energy of deformation became $3.0(1.1) \mathrm{mJ} \mathrm{mm}^{-3}$. The energetic coefficient of restitution, $e_{*}$, did not vary with site over the femoral head $(P=0.57)$ and the mean value was $0.502(0.066)$, showing that only one-quarter of the energy stored $\left(e_{*}^{2}\right)$ was returned elastically, the remainder being dissipated.

\subsection{Mass and composition}

Following impact, there was no detectable mass loss within the resolution of the balance $(0.01$ mg). On replacing in PBS there was a small gain in mass of $1.98 \%$ [1.37 \%, $2.98 \%$ ] after 24 h compared with the initial equilibrium mass.

Biochemical analysis showed the overall composition to be 70.9 (2.6) \% water, 11.1 (2.9) \% collagen, 5.4 (1.3) \% GAG, leaving $12.6 \%$ of the mass not accounted for by these measurements. The water and GAG content of samples varied with site over the femoral head but no variation was found in collagen content (Table 2). ANOVA showed significant differences for percentage wet weight GAG over the femoral head $(P<0.001)$ and pair-wise comparisons showed the inferior region to have a lower GAG content compared with all other sites except the lateral anterior (A2) and lateral posterior (P2) sites. There were no significant differences between any of the other sites. The percentage water content of the samples also showed some significant differences $(P=0.002)$ between sites with the inferior region and 
lateral anterior region (A2) having a higher water content than the medial superior-posterior region (SP1).

Table 2. Cartilage composition. Mean (SD) values for collagen, GAG and water contents expressed in terms of wet mass of tissue for samples at sites over the human femoral head described in Figure 1.

\begin{tabular}{|l|l|l|l|}
\hline Site & Collagen $/ \%$ & \multicolumn{1}{|c|}{ GAG $/ \%$} & Water $/ \%$ \\
\hline S1 & $9.3(2.5)$ & $5.8(1.1)$ & $70.2(2.6)$ \\
SP1 & $10.3(2.2)$ & $5.7(1.7)$ & $69.6(2.8)$ \\
SP2 & $10.9(2.6)$ & $5.5(0.9)$ & $70.8(2.5)$ \\
SA1 & $9.1(2.1)$ & $5.7(0.8)$ & $69.9(2.0)$ \\
SA2 & $11.0(2.7)$ & $5.5(1.3)$ & $71.6(2.3)$ \\
P1 & $10.1(1.7)$ & $6.1(1.3)$ & $70.1(1.7)$ \\
P2 & $11.4(2.5)$ & $4.9(0.8)$ & $72.1(2.1)$ \\
A1 & $8.2(2.8)$ & $5.8(1.2)$ & $70.2(3.2)$ \\
A2 & $11.2(3.4)$ & $5.0(0.8)$ & $73.4(2.1)$ \\
I1 & $12.1(2.6)$ & $3.6(1.5)$ & $72.9(3.0)$ \\
\hline
\end{tabular}

Correlations between composition and dynamic and quasi-static moduli (Table 3) showed that moduli were more strongly associated with collagen and water contents than with GAG content. The correlations, however, were reversed between quasistatic and dynamic moduli. Water and GAG contents were negatively correlated $(R=-0.35, P<0.001)$. The energy of deformation was significantly correlated only with water and collagen contents $(P<0.001)$. 
Table 3. Relationships between moduli and composition. Correlation coefficients, R, and significance values, $\mathrm{P}$, for pair-wise comparisons of mechanical and fractional compositional properties calculated using Spearman's rank order correlation.

\begin{tabular}{lcccc}
\hline & & Water\% & GAG\% & Collagen\% \\
\hline$E_{\text {dyn }}$ & $R$ & 0.33 & -0.23 & 0.49 \\
& $P$ & 0.002 & 0.019 & $<0.001$ \\
$E_{\text {slow }}$ & $R$ & -0.43 & 0.27 & -0.33 \\
& $P$ & 0.001 & 0.037 & 0.012
\end{tabular}

\section{Discussion}

In this study we attempt to relate measures of mechanical properties at slow and impact loading rates with measurements of gross composition in elderly human articular cartilage. Many studies have been done using animal tissue and slow rates of loading, fewer using impact loading and fewer still exploring relationships between mechanical and compositional measurements for which, currently, there is no conceptual framework. Tissue from the elderly is of particular interest as it is more likely to suffer degeneration in diseases such as OA, although for the individuals in this study it has survived with no apparent loss of integrity. The age of the donors means that the tissue often appears slightly matt, unlike the shiny surface of young tissue, but care was taken to exclude tissue showing any signs 'fraying' or overt damage that could be seen by visual inspection. Despite the limitations of this study we extend previous data to incorporate features of dynamic loading such as a dynamic modulus and the coefficient of restitution.

The mean peak dynamic modulus measured at each site over the femoral head was between 20 and 60 times greater than that measured at the same site using slow rates of loading. 
Comparing the moduli at different loading rates at the same stress of $0.1 \mathrm{MPa}$, perhaps a fairer comparison, indicates that the dynamic modulus is very similar to that measured during slow loading, although measuring the dynamic modulus at this stress was difficult as this point lies within the first few data points of the stress-strain curve (Fig. 2). At fast rates of loading, however, the modulus rapidly increases to reduce the strain sustained by the tissue and a nonlinear model is almost certainly required to understand the behaviour of the tissue [30]. It is the modulus measured using conventional 'slow' loading that is traditionally quoted for cartilage and used in modelling. Although impact loading may be deemed too serious a challenge, only rarely encountered in normal living, it does represent the upper limits of what the tissue may have to withstand. Although much of the variation in dynamic modulus might be due to different rates of loading resulting from the different tissue thicknesses this does reflect the situation in vivo where tissue thickness is an important factor; soft, deep cushions are better at attenuating load than thin, stiff ones. When it comes to developing tissue engineering approaches for cartilage repair it does indicate that typically quoted values of $\sim 1$ MPa are the lower bound of what needs to be achieved to provide adequate mechanical competency.

The impact energy used here was chosen to cause minimal damage to the tissue. We have shown this previously using histology $[5,15,24]$ and similar results were found here with no evidence of fissuring in histological sections of a selection of samples (data not shown). Unlike testing using a standard testing machine, during impact loading there is no control over the peak load or deformation so constant energy was chosen as the governing factor. This means that the maximum stress and strain, and stress- and strain-rates, experienced by the sample can vary over a considerable range of values. A theoretical analysis of constant energy testing shows that the applied stress depends on both the modulus and the thickness of 
the sample [31] and the strain-rate clearly depends on the sample thickness. The data showed that both the dynamic and the quasi-static moduli were found to be correlated with tissue thickness, although in opposite ways.

The thickness of the cartilage over the femoral head, thicker superiorly and thinner on the inferior aspect, and the variation in composition were consistent with results from previous studies [32-34]. The mean thickness is small, but typical for elderly tissue, and non-contact measurement ensures no compression occurs during measurement. The pattern for the quasistatic modulus generally followed that of the thickness, with the thickest and stiffest cartilage being found on the superior aspect of the femoral head [33,35,36]. A partial explanation of this may be provided by the analysis of Eberhardt et al. [37] who related stresses in the tissue to the ratio of contact area to thickness. Increasing thickness, leading to a smaller ratio, resulted in greater stresses for a given strain and, hence, a greater modulus. Dynamic measurements showed a reversal of this pattern with the stiffest cartilage being found in the inferior region and peripheral areas of the femoral head, where the cartilage was thinnest. Adjusting the measurements as if to unit thickness, however, removed the site variation indicating that thickness, rather than composition, has the main effect on the measured values of $E_{d y n}$. Unsurprisingly, the thicker and softer the cartilage, the more energy is absorbed and this provides a mechanism to absorb energy in the most heavily loaded regions to prevent crack formation and propagation. Curiously, there was no significant sitevariation of the strain in the tissue at the point of maximum modulus and the variation in this value measured during rapid loading was considerably less than the variation in either modulus or thickness. A six-fold variation between smallest and largest $E_{d y n}$, ranging from 25 MPa to $150 \mathrm{MPa}$, and thickness, between 0.42 and $2.57 \mathrm{~mm}$, resulted in only a 2.5-fold variation in strain at peak modulus. It has been suggested that the chondrocytes can optimize 
the tissue modulus in order to produce a strain environment conducive to their survival [38] and perhaps this result reflects a consequence of that hypothesis; composition (controlling modulus) and thickness being jointly regulated to try to ensure strains are maintained within certain bounds.

Water flow has less time to occur during an impact, which typically lasts about $0.5 \mathrm{~ms}$, and the absence of any measurable loss of mass during impact supports our previous studies that no water is expelled at high rates of loading [15], despite the large strains developed. Studies using rapid, although not true impact, loading have reported fluid losses that decreased with increasing loading rate and were a few percent at the highest rates of loading [14]. Previously, we, and others, have suggested that cartilage subjected to impact loading might be expected to become more elastic $[15,21]$ on the premise that water would not have time to flow and would generate an increased hydrostatic pressure. However, experiment shows that this is not the case. The impact remains viscoelastic, the coefficient of restitution decreases with increasing impact velocity and, in a separate study, we found that a non-linear modification of a Maxwell-like viscoelastic model was needed to fit the data [30]. Given the high water content, we hypothesize that to understand the mechanical behaviour of cartilage over a wide range of loading rates it might be better considered as a hydrogel than a porous solid. Although no visible damage occurred, the small mass increase following re-immersion in fluid indicates that some internal or structural damage has occurred enabling the tissue to swell slightly.

A number of factors complicate the testing of articular cartilage in practice; friction between the sample and the loading platens leads to an overestimation of the modulus [39-41] and the small aspect ratio (height/diameter) of the samples make the specimen-platen considerations even more important. Because the samples had the same diameter may mean that these effects 
will be similar across all the samples. The testing chosen was unconfined and slow testing was done to a fixed stress whereas impact was to a constant energy. The samples were also taken from elderly individuals and extrapolating to younger tissue is not possible. These issues make measurement of absolute material properties difficult but may not seriously impair the comparative nature of the tests in this study as all samples were subjected to the same testing regimes.

The relationship between composition and mechanical properties depends on many factors, including species [42], joint and site within a joint [35,43] and testing regime (unconfined or confined compression, tension, shear etc.). Early studies suggested that cartilage compressive properties are related most strongly to water and GAG content [44]; but more recent studies have indicated a more complicated relationship. For example, in earlier studies, water, sulphated GAG and a measure of collagen content were required to account for over $80 \%$ of the variance in the equilibrium compressive modulus [45], and in studies of bovine carpometacarpal joint cartilage we found no site variation in mechanical properties despite a variation in composition similar to that found over human femoral heads [12]. The large deformations experienced by the tissue during an impact [30], and the lack of time for fluid to flow, are likely to underlie the dependency of dynamic modulus on collagen and water contents rather than GAG content. The correlation coefficients, however, indicate that only about $25 \%$ of the variance in dynamic modulus is explained by the composition as measured. It is not clear why the collagen content we measured was lower than previous studies, which have suggested about $16 \%$ in femoral head cartilage from similar-aged individuals [46]. The site variation is similar to that found previously and a systematic difference should not affect the correlations. Using chondroitin sulphate as the standard will underestimate GAG content as it will not account for keratan sulphate or hyaluronon. We chose this, however, as 
chondroitin sulphate will be the largest contributor to fixed-charge density and swelling pressure, and is present in greater molar quantities. Other proteins, e.g. the core protein of aggrecan, and non-sulphated polysaccharides such as hyaluronan will comprise the mass not accounted for and it is possible that these could explain more of the variance. Modelling studies [47] have supported the idea that interactions between collagen and GAG are also important [11,48], and if the tissue is considered as a fibre-composite material then these interactions, which govern the way stress is transferred from the matrix to the fibres, are crucial for determining the material properties [11].

Most previous studies of cartilage mechanical behaviour, and especially those trying to relate function to composition, have been done at slow rates of loading. This study extends these into physiological and pathological rates of loading. Elderly human articular cartilage is a resilient tissue whose modulus increases non-linearly with rate of loading. During impact loading, the peak modulus reaches values approaching two orders of magnitude larger than those measured during quasi-static loading. The coefficient of restitution varies little over the femoral head and the dynamic modulus is smallest where the tissue is thickest, providing an energy absorbing mechanism. Composition alone is a poor predictor of modulus at both slow and fast loading rates. Fibre-reinforced composites depend crucially on the interactions between the fibres and their surrounding matrix and it is in understanding and controlling these that much work needs to be done.

\section{Acknowledgments}

We thank the Orthopaedic Surgeons of NHS Grampian for kindly providing us with tissue donated by their patients. 


\section{Competing interests}

The authors have no competing interests to declare.

\section{Funding}

This study was funded by a project grant from Arthritis Research UK (formerly the Arthritis Research Campaign) Grant number A0542 that provided a salary for LVB. The funder had no involvement in the design or execution of the study or the publication of results.

\section{Ethical approval}

Approval for the use of discarded tissue removed during the course of standard surgery was given by the Grampian Health Board and University of Aberdeen Joint Ethical Committee, reference number 9800033. 


\section{References}

[1] Martin JA, Brown T, Heiner A, Buckwalter JA. Post-traumatic osteoarthritis: The role of accelerated chondrocyte senescence. Biorheology 2004;41:479-91.

[2] Kim HT, Lo MY, Pillarisetty R. Chondrocyte apoptosis following intraarticular fracture in humans. Osteoarthritis Cartilage 2002;10:747-9.

[3] Little CJ, Bawolin NK, Chen X. Mechanical properties of natural cartilage and tissueengineered constructs. Tissue Eng Part B Rev 2011;17:213-27.

[4] Aspden RM, Jeffrey JE, Burgin LV. Impact loading: physiological or pathological? Osteoarthritis Cart 2002;10:588-9.

[5] Jeffrey JE, Aspden RM. The biophysical effects of a single impact load on human and bovine articular cartilage. Proc Instn Mech Engrs [H], J Eng Med 2006;220:677-86.

[6] Plumb MS, Aspden RM. The response of elderly human articular cartilage to mechanical stimuli in vitro. Osteoarthritis Cart 2005;13:1084-91.

[7] Pedersen DR, Goetz JE, Kurriger GL, Martin JA. Comparative digital cartilage histology for human and common osteoarthritis models. Orthopedic Research and Reviews 2013;5:13-20.

[8] Nissi MJ, Rieppo J, Toyras J, Laasanen MS, Kiviranta I, Nieminen MT, et al. Estimation of mechanical properties of articular cartilage with MRI - dGEMRIC, T(2) and T(1) imaging in different species with variable stages of maturation. Osteoarthritis Cartilage 2007;15:1141-8.

[9] Demarteau O, Pillet L, Inaebnit A, Borens O, Quinn TM. Biomechanical characterization and in vitro mechanical injury of elderly human femoral head 
cartilage: comparison to adult bovine humeral head cartilage. Osteoarthritis Cart 2006;14:589-96.

[10] Hukins DWL, Aspden RM. Composition and properties of connective tissues. Trends Biochem Sci 1985;10:260-4.

[11] Aspden RM. Fibre reinforcing by collagen in cartilage and soft connective tissues. Proc R Soc Lond B-Biol Sci 1994;258:195-200.

[12] Lewis RJ, MacFarland AK, Anandavijayan S, Aspden RM. Site variation of material properties and metabolic activity of articular cartilage from the bovine carpometacarpal joint. Osteoarthritis Cart 1998;6:383-92.

[13] Milentijevic D, Helfet DL, Torzilli PA. Influence of stress magnitude on water loss and chondrocyte viability in impacted articular cartilage. J Biomech Eng 2003;125:594-601.

[14] Milentijevic D, Torzilli PA. Influence of stress rate on water loss, matrix deformation and chondrocyte viability in impacted articular cartilage. J Biomech 2005;38:493-502.

[15] Burgin LV, Aspden RM. Impact testing to determine the mechanical properties of articular cartilage in isolation and on bone. J Mater Sci :Mater Med 2008;19:703-11.

[16] Radin EL, Paul IL, Lowy M. A comparison of the dynamic force transmitting properties of subchondral bone and articular cartilage. J Bone Joint Surg 1970;52A:444-56.

[17] Radin EL, Paul IL. Does cartilage compliance reduce skeletal impact loads? The relative force-attenuating properties of articular cartilage, synovial fluid, periarticular soft tissues and bone. Arthritis Rheum 1970;13:139-44. 
[18] Finlay JB, Repo RU. Impact characteristics of articular cartilage. ISA Transactions $1978 ; 17: 29-34$.

[19] Finlay JB, Repo RU. Impact characteristics of articular cartilage. Biomed Sci Instrum $1977 ; 13: 47-50$

[20] Natoli RM, Athanasiou KA. Traumatic loading of articular cartilage: Mechanical and biological responses and post-injury treatment. Biorheology 2009;46:451-85.

[21] Oloyede A, Flachsmann R, Broom ND. The dramatic influence of loading velocity on the compressive response of articular cartilage. Connect Tissue Res 1992;27:211-4.

[22] Verteramo A, Seedhom BB. Effect of a single impact loading on the structure and mechanical properties of articular cartilage. J Biomech 2007;40:3580-9.

[23] Brown TD, Shaw DT. In vitro contact stress distributions in the natural human hip. J Biomech 1983;16:373-84.

[24] Jeffrey JE, Gregory DW, Aspden RM. Matrix damage and chondrocyte viability following a single impact load on articular cartilage. Arch Biochem Biophys 1995;322:87-96

[25] Burgin LV, Aspden RM. A drop tower for controlled impact testing of biological tissues. Med Eng Phys 2007;29:525-30.

[26] Stronge WJ. Impact Mechanics. Cambridge: Cambridge University Press; 2000.

[27] Stone JE, Akhtar N, Botchway S, Pennock CA. Interaction of 1,9-dimethylmethylene blue with glycosaminoglycans. Ann Clin Biochemistry 1994;31:147-52. 
[28] Woessner JF. Determination of hydroxyproline in connective tissues. In: Hall DA, editor. The methodology of connective tissue research.Oxford: Joynsson-Bruvvers Ltd.; 1976. p. 227-33.

[29] Ignat'eva NY, Danilov NA, Averkiev SV, Obrezkova MV, Lunin VV, Sobol' EN. Determination of hydroxyproline in tissues and the evaluation of the collagen content of the tissues. Journal of Analytical Chemistry 2007;62:51-7.

[30] Edelsten L, Jeffrey JE, Burgin LV, Aspden RM. Viscoelastic deformation of articular cartilage during impact loading. Soft Matter 2010;6:5206-12.

[31] Pytel A, Singer FL. Strength of Materials. 4 ed. New York: Harper \& Row; 1987.

[32] Venn MF. Chemical composition of human femoral and head cartilage: influence of topographical position and fibrillation. Ann Rheum Dis 1979;38:57-62.

[33] Athanasiou KA, Agarwal A, Dzida FJ. Comparative study of the intrinsic mechanical properties of the human acetabular and femoral head cartilage. J Orthop Res 1994;12:340-9.

[34] Shepherd DE, Seedhom BB. Thickness of human articular cartilage in joints of the lower limb. Ann Rheum Dis 1999;58:27-34.

[35] Shepherd DE, Seedhom BB. The 'instantaneous' compressive modulus of human articular cartilage in joints of the lower limb. Rheumatology 1999;38:124-32.

[36] Kempson GE, Spivey CJ, Swanson SA, Freeman MA. Patterns of cartilage stiffness on normal and degenerate human femoral heads. J Biomech 1971;4:597-609. 
[37] Eberhardt AW, Keer LM, Lewis JL, Vithoontien V. An analytical model of joint contact. J Biomech Eng 1990;112:407-13.

[38] Barker MK, Seedhom BB. The relationship of the compressive modulus of articular cartilage with its deformation response to cyclic loading: does cartilage optimize its modulus so as to minimize the strains arising in it due to the prevalent loading regime? Rheumatology 2001;40:274-84.

[39] Aspden RM. Constraining the lateral dimensions of uniaxially loaded materials increases the calculated strength and stiffness: application to muscle and bone. J Mater Sci :Mater Med 1990;1:100-4.

[40] Aspden RM. The effect of boundary conditions on the results of mechanical tests. J Biomech 1990;23:623.

[41] Odgaard A, Linde F. The underestimation of Young's modulus in compressive testing of cancellous bone specimens. J Biomech 1991;24:691-8.

[42] Athanasiou KA, Agarwal A, Muffoletto A, Dzida FJ, Constantinides G, Clem M. Biomechanical properties of hip cartilage in experimental animal models. Clin Orthop $1995 ; 254-66$.

[43] Froimson MI, Ratcliffe A, Gardner TR, Mow VC. Differences in patellofemoral joint cartilage material properties and their significance to the etiology of cartilage surface fibrillation. Osteoarthritis Cart 1997;5:377-86.

[44] Kempson GE, Muir H, Freeman MAR, Swanson SAV. Correlations between the compressive stiffness and chemical constituents of human articular cartilage. Biochim Biophys Acta 1970;215:70-7. 
[45] Treppo S, Koepp H, Quan EC, Cole AA, Kuettner KE, Grodzinsky AJ. Comparison of biomechanical and biochemical properties of cartilage from human knee and ankle pairs. J Orthop Res 2000;18:739-48.

[46] Maroudas A, Bayliss MT, Venn MF. Further studies on the composition of human femoral head cartilage. Ann Rheum Dis 1980;39:514-23.

[47] Thomas GC, Asanbaeva A, Vena P, Sah RL, Klisch SM. A nonlinear constituent based viscoelastic model for articular cartilage and analysis of tissue remodeling due to altered glycosaminoglycan-collagen interactions. J Biomech Eng 2009;131:101002.

[48] Zhu W, Iatridis JC, Hlibczuk V, Ratcliffe A, Mow VC. Determination of collagenproteoglycan interactions in vitro. J Biomech 1996;29:773-83. 